\title{
Zinc finger protein 185 is a liver metastasis-associated factor in colon cancer patients
}

\author{
DAISUKE FURUKAWA ${ }^{1}$, TSUYOSHI CHIJIWA ${ }^{2}$, MASAHIRO MATSUYAMA ${ }^{1}$, MASAYA MUKAI ${ }^{3}$, \\ EI-ICHI MATSUO ${ }^{4}$, OSAMU NISHIMURA ${ }^{5}$, KENJI KAWAI $^{6}$, HIROSHI SUEMIZU ${ }^{2}$, NOBUYOSHI HIRAOKA $^{7}$, \\ TOSHIO NAKAGOHRI ${ }^{1}$, SEIEI YASUDA ${ }^{1}$ and MASATO NAKAMURA ${ }^{8}$ \\ ${ }^{1}$ Department of Surgery, Tokai University School of Medicine, Isehara, Kanagawa 259-1193; \\ ${ }^{2}$ Laboratory Animal Research Department, Central Institute for Experimental Animals, Kawasaki, Kanagawa 210-0821; \\ ${ }^{3}$ Department of Surgery, Tokai University Hachioji Hospital, Hachioji, Tokyo 192-0032; \\ ${ }^{4}$ Global Application Development Center, Analytical and Measuring Instruments Division, Shimadzu Corporation, \\ Kyoto 604-8511; ${ }^{5}$ The Integrated Center for Mass Spectrometry, Graduate School of Medicine, Kobe University, \\ Kobe, Hyogo 650-0017; ${ }^{6}$ Pathological Analysis Center, Central Institute for Experimental Animals, Kawasaki, \\ Kanagawa 210-0821; ${ }^{7}$ Division of Molecular Pathology, National Cancer Center Research Institute, Tokyo 104-0045; \\ ${ }^{8}$ Department of Regenerative Medicine, Tokai University School of Medicine, Isehara, Kanagawa 259-1193, Japan
}

Received February 20, 2014; Accepted March 25, 2014

DOI: $10.3892 /$ mco.2014.298

\begin{abstract}
LIM domain proteins are involved in several fundamental biological processes, including cell lineage specification, cytoskeleton organization and organ development. Zinc finger protein 185 (ZNF185) is one of the LIM domain proteins considered to be involved in the regulation of cellular differentiation and/or proliferation. However, the detailed functions and properties of ZNF185 in the multistep process of cancer biology have not yet been elucidated. In this study, we analyzed the association between ZNF185 and the clinicopathological characteristics of colon cancer, such as patient age and gender, histological type, lymphatic and venous involvement, $\mathrm{T}$ and $\mathrm{N}$ status, liver metastasis and stage. ZNF185 protein expression was immunohistochemically analyzed and ZNF185 was detected in the cancer cells of 78 of the 87 colon cancer patients. The correlation between ZNF185 and histological type was significant $(\mathrm{P}=0.010$, G-test). ZNF185 expression was also significantly correlated with liver metastasis $(\mathrm{P}=0.030, \mathrm{G}$-test $)$. A multivariate analysis using the Cox proportional hazards model was performed among cause-specific survival rate, ZNF185 expression and clinicopathological characteristics. Histological type, liver metastasis and ZNF185 expression were found to be independent prognostic indicators $(\mathrm{P}=0.028, \mathrm{P}<0.0001$ and $\mathrm{P}=0.036$,
\end{abstract}

Correspondence to: Professor Masato Nakamura, Department of Regenerative Medicine, Tokai University School of Medicine, 143 Shimokasuya, Isehara, Kanagawa 259-1193, Japan

E-mail: mnakamur@is.icc.u-tokai.ac.jp

Key words: zinc finger protein 185 , colon cancer, liver metastasis, prognosis respectively). Therefore, ZNF185 expression was found to be an independent indicator of liver metastasis and prognosis in patients with colon cancer.

\section{Introduction}

Colorectal cancer is among the three leading causes of cancer-related mortality worldwide. Approximately 50\% of patients with colon cancer, the predominant type of colorectal cancer, develop liver metastasis, which is considered to be the main cause of death from advanced-stage colon cancer (1). Therefore, it is crucial to elucidate the biological mechanisms underlying liver metastasis of colon cancer and accelerate the development of new treatment strategies.

The liver is the most common site for metastasis from colon and pancreatic cancer (2). Hepatectomy is a potentially curative treatment option for liver metastasis from colon cancer; however, liver metastasis from pancreatic cancer is not considered an indication for surgical treatment (3). Similarities or differences in the biology of liver metastasis between colon and pancreatic cancer remain to be elucidated. We previously established and investigated the highly liver-metastatic human colorectal cancer cell sublines SW48LM2 and LM-BxPC-3, through the serial intrasplenic transfer of hepatic tumor foci formed by parental SW48 colon cancer cells and BxPC-3 pancreatic cancer cells in NOD/Shi-scid/IL-2R $\gamma^{\text {null }}$ mice (4-6). We then performed a quantitative proteome analysis utilizing these established cell lines by our original method (7). The comparison of cellular protein abundance between a pair of 'highly liver-metastatic' cells and its parental cells revealed a series of metastasis-related proteins. In order to identify more universal metastasis-related proteins, we subsequently selected 11 proteins commonly detected among two pairs, i.e., the BxPC-3 and the SW48 subline pairs (unpublished data). These proteins are expected to be good biomarker candidates 
and/or plausible causal factors for cancer metastasis. Zinc finger protein 185 (ZNF185) is one of these 11 proteins thus selected.

ZNF185 belongs to the family of LIM domain proteins and contains one LIM zinc-binding domain at the $\mathrm{COOH}$-terminus and an actin-targeting domain (ATD) at the $\mathrm{NH}_{2}$-terminus. The LIM domain is a cysteine- and histidine-rich double zinc-finger motif named after the three homeodomain proteins: Lin-1 1, Isl-1 and Mec-3 (8-10). The LIM domain is present in a wide range of proteins whose functions include a number of fundamental biological processes, such as cell lineage specification, cytoskeleton organization and organ development (11). Whereas the Zinc finger motif contains the typical DNA binding structures, there is little evidence to support the observation that LIM domains may directly bind DNA (12). LIM domain proteins were found to be distributed in the cytoplasm or nucleus of cells and perform regulatory functions through protein:protein interactions rather than direct interactions with DNA $(13,14)$. ZNF185 is located on chromosome Xq28 and is expressed in the kidney, prostate, pancreas, blood, placenta and ovary, but not in the liver (15). ZNF185 may be involved in regulating cellular differentiation and/or proliferation $(16,17)$. Certain LIM domain-containing proteins were previously shown to be involved in carcinogenic processes (18-27). In basic research on prostate cancer, craniocervical squamous cell carcinoma and non-small-cell lung cancer, the underexpression of ZNF185 mRNA in tumoral tissue was compared to that in matched normal tissue $(16,28,29)$. However, the detailed properties and functions of ZNF185 in the multistep process of tumor invasion have not been investigated in detail (12). The clinical behavior of ZNF185 also remains unknown in relation to the prognosis or treatment of various cancers.

In this study, we investigated the expression level of ZNF185 using immunohistochemistry in 87 cases of colon cancer obtained by complete surgical resection. We also discussed the association between prognosis and the clinical significance of ZNF185 expression.

\section{Materials and methods}

Patients. A total of 87 colon cancer specimens were obtained from the surgical specimens of patients with informed consent between April, 2002 and May, 2005. This study has been approved by the Institutional Research Review Board of Tokai University. The tissues were immediately fixed in $40 \%$ formaldehyde. The surgical specimens were also processed for routine histopathological analysis.

The patient sample included 48 men and 39 women, with a mean age of $68.30 \pm 9.26$ years. Well-differentiated adenocarcinomas were found in 60 patients, moderately differentiated adenocarcinomas in 21 , poorly differentiated adenocarcinomas in 2 and mucinous adenocarcinomas in 4 patients. The tumors were clinically staged according to the Union for International Cancer Control TNM system. The tumor status was T1 in 5 patients, T2 in 11, T3 in 55 and T4 in 16 patients. A total of 46 patients had lymph node metastasis (N1) and 18 patients had distant metastasis (M1). Lymphatic and venous involvement was found in 74 and 46 patients, respectively. A total of 19 patients had liver metastasis, including 10 synchronous liver metastasis patients. The pathological stages were as follows: stage I, 9 patients; stage II, 30 patients; stage III, 30 patients; and stage IV, 18 patients.

Immunohistochemical (IHC) analysis. Formalin-fixed, paraffin-embedded tissue sections of the tumor samples were analyzed. The paraffin-embedded sections were deparaffinized and stained using the streptavidin-biotinperoxidase complex method. Rabbit antibodies specific to ZNF185, activated RNA polymerase II transcriptional coactivator p15 (SUB1), $\beta$ - $N$-acetylhexosaminidase A (HEXA), general transcription initiation factor IIF $\alpha$ subunit (GTF2F1), actinin $\alpha 4$ (ACTN4) and interleukin enhancerbinding factor 3 (ILF3) were purchased from Sigma-Aldrich Co. (St. Louis, MO, USA). Rabbit anti-glucosidase 2 subunit $\beta$ (Gluco) and clathrin heavy chain (CLTC) antibodies were purchased from Abcam ${ }^{\circledR}$ (Cambridge, UK). Briefly, the sections were incubated in $0.3 \% \mathrm{H}_{2} \mathrm{O}_{2}$ in methanol, washed in phosphate-buffered saline (PBS) and non-specific protein binding was blocked with normal rabbit serum (Nichirei, Tokyo, Japan). The sections were then incubated overnight in a humid chamber at $4^{\circ} \mathrm{C}$, with affinity purified antibodies diluted in PBS, as recommended by the manufacturers. Following three PBS washes, the sections were incubated with peroxidase-labeled polymer conjugated rabbit anti-goat antibody (Histofine Simple Stain Max PO; Nichirei). The amplified immune products were visualized using a 3,3'-diaminobenzidine tetrahydrochloride reaction.

Statistical analysis. Statistical comparisons of data sets were performed by non-parametric analysis using the Mann-Whitney U test. The G-test (likelihood ratio Chi-square test) was applied for comparisons between group frequencies. On multivariate analyses of the cause-specific survival rate, the Cox proportional hazards model was used. Data are presented as means \pm standard deviation. The analyses were performed using JMP version 8 software (SAS Institute Inc., Cary, NC, USA). $\mathrm{P}<0.05$ was considered to indicate a statistically significant difference.

\section{Results}

Identification of ZNF185 as a liver metastasis-associated factor. We selected 8 proteins for the IHC staining experiment among the 11 liver metastasis-associated proteins identified and selected by quantitative proteome studies (unpublished data). The expression of each protein in surgically resected specimens from colon cancer was evaluated by IHC staining. Statistical analyses were performed between expression of the ZNF185, SUB1 and HEXA proteins and liver metastasis in 87 colon cancer cases. A significant correlation was only observed for ZNF185 expression, whereas the correlations were not significant for the expression of SUB1 and HEXA ( $\mathrm{P}=0.030$, G-test) (Table I). Specific expression of the GTF2F1, ACTN4, ILF3, CLTC and Gluco proteins could not be detected using standard IHC procedures.

ZNF185 expression and clinicopathological characteristics. ZNF185 expression was observed in 78 of the 87 colon cancer cases (Fig. 1). A significant difference was observed between histological type and ZNF185 expression $(\mathrm{P}=0.010$, 

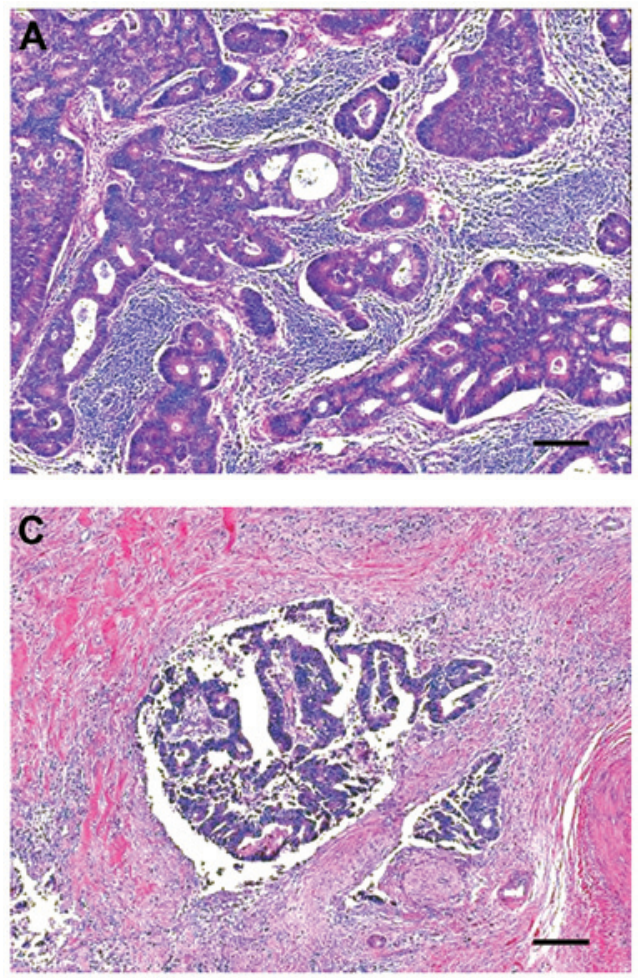
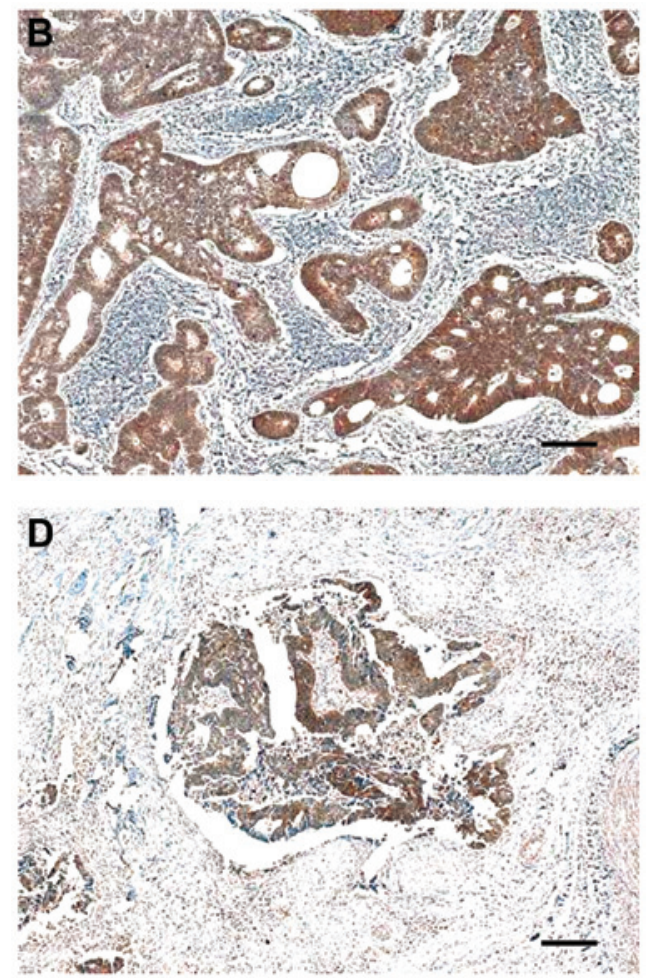

Figure 1. Zinc finger protein 185 (ZNF185) expression in human colon cancer. Colon cancer cells (A and C) exhibit strongly positive cytoplasmic staining for ZNF185 (B and D). Bar, $100 \mu \mathrm{m}$.

Table I. Expression of candidate molecules in liver metastasis from colon cancer.

\begin{tabular}{lccc}
\hline \multirow{2}{*}{$\begin{array}{l}\text { Candidate } \\
\text { molecules (n) }\end{array}$} & \multicolumn{2}{c}{ Total liver metastasis } & \\
\cline { 2 - 3 } & Positive (19) & Negative (68) & P-value \\
\hline ZNF185 & & & \\
$\quad$ Positive (78) & 19 & 59 & $0.030^{\mathrm{a}}$ \\
$\quad$ Negative (9) & 0 & 9 & \\
HEXA & & & \\
Positive (69) & 14 & 55 & 0.347 \\
$\quad$ Negative (18) & 5 & 13 & \\
SUB1 & & & \\
Positive (84) & 19 & 65 & 0.473 \\
Negative (3) & 0 & 3 & \\
\hline
\end{tabular}

G-test; ${ }^{\text {P }}<0.05$. ZNF185, zinc finger protein 185 .

G-test). Other clinicopathological correlations, including synchronous liver metastasis, were not significant (Table II). The mean age of ZNF185-positive and -negative patients was $67.67 \pm 9.07$ and $73.78 \pm 9.61$ years, respectively $(\mathrm{P}=0.071$, Mann-Whitney U test).

Correlations between prognosis and ZNF185 expression in colon cancer. We analyzed the correlations among cause-specific survival rate, ZNF185 expression and clinicopathological characteristics, such as patient age and gender, histological type, lymphatic and venous involvement, $\mathrm{T}$ and
$\mathrm{N}$ status, synchronous liver metastasis and stage, using the Cox proportional hazards model. The multivariate analyses identified histological type, synchronous liver metastasis and ZNF185 expression as independent prognostic indicators $(\mathrm{P}=0.029, \mathrm{P}<0.0001$ and $\mathrm{P}=0.020$, respectively) (Table III).

\section{Discussion}

In this study, we identified ZNF185 as a significant liver metastasis-associated factor in colon cancer. To the best of our knowledge, this is the first study to investigate the association between ZNF185 and the clinical characteristics of cancer. ZNF185 expression in colon cancer was found to be an indicator of liver metastasis, as well as an independent prognostic indicator. The histological type and synchronous liver metastasis were found to significantly affect the prognosis of colon cancer patients.

ZNF185 belongs to the LIM domain protein family containing two zinc-finger motifs in the C-terminus, classified as group 3 (12,30). ZNF185 is located on chromosome Xq28 and is expressed in the kidney, prostate, pancreas, blood, placenta and ovary, but not in the liver (15). The complete ZNF185 gene was originally cloned from normal human prostate tissue by Zhang et al (17). The expression and localization of ZNF185 in prostate cancer cells and fibroblasts revealed that, in addition to F-actin stress fibers, ZNF185 localized to several other cytoskeleton-related areas, including focal adhesions and filopodia/ lamellipodia. ZNF185 was also shown to contain an ATD in the $\mathrm{N}$-terminal region and binds to F-actin directly through the ATD, but not the LIM domains (17). Thus, ZNF185 interacts with F-actin and focal adhesion components. Further studies, focused on identifying proteins interacting with the other domains of 
Table II. ZNF185 expression in colon cancer.

\begin{tabular}{|c|c|c|c|}
\hline \multirow{2}{*}{$\begin{array}{l}\text { Clinicopathological } \\
\text { characteristics }(\mathrm{n})\end{array}$} & \multicolumn{2}{|c|}{ ZNF185 expression } & \multirow[b]{2}{*}{ P-value } \\
\hline & $(+)$ & $(-)$ & \\
\hline \multicolumn{4}{|l|}{ Gender } \\
\hline Male (48) & 44 & 4 & 0.496 \\
\hline Female (39) & 34 & 5 & \\
\hline \multicolumn{4}{|l|}{ Histological type } \\
\hline $\begin{array}{l}\text { Well differentiated } \\
\text { adenocarcinoma }(60)\end{array}$ & 56 & 4 & $0.010^{\mathrm{a}}$ \\
\hline $\begin{array}{l}\text { Moderately differentiated } \\
\text { adenocarcinoma (21) }\end{array}$ & 18 & 3 & \\
\hline $\begin{array}{l}\text { Poorly differentiated } \\
\text { adenocarcinoma (2) }\end{array}$ & 0 & 2 & \\
\hline Mucinous adenocarcinoma (4) & 4 & 0 & \\
\hline \multicolumn{4}{|l|}{ T status } \\
\hline $\mathrm{T} 1(5)$ & 5 & 0 & 0.599 \\
\hline $\mathrm{T} 2(11)$ & 9 & 2 & \\
\hline T3 (55) & 50 & 5 & \\
\hline $\mathrm{T} 4$ (16) & 14 & 2 & \\
\hline \multicolumn{4}{|l|}{ N status } \\
\hline N0 (41) & 35 & 6 & 0.213 \\
\hline N1 (46) & 43 & 3 & \\
\hline \multicolumn{4}{|l|}{ M status } \\
\hline M0 (69) & 61 & 8 & 0.424 \\
\hline M1 (18) & 17 & 1 & \\
\hline \multicolumn{4}{|l|}{ Lymphatic involvement } \\
\hline Positive (74) & 67 & 7 & 0.538 \\
\hline Negative (13) & 11 & 2 & \\
\hline \multicolumn{4}{|l|}{ Venous involvement } \\
\hline Positive (46) & 41 & 5 & 0.835 \\
\hline Negative (41) & 37 & 4 & \\
\hline \multicolumn{4}{|l|}{ Synchronous liver metastasis } \\
\hline Positive (10) & 10 & 0 & 0.127 \\
\hline Negative (77) & 68 & 9 & \\
\hline \multicolumn{4}{|l|}{ Stage } \\
\hline $\mathrm{I}(9)$ & 7 & 2 & 0.502 \\
\hline II (30) & 26 & 4 & \\
\hline III (30) & 28 & 2 & \\
\hline IV (18) & 17 & 1 & \\
\hline
\end{tabular}

G-test; ${ }^{a} \mathrm{P}<0.05 . \mathrm{ZNF} 185$, zinc finger protein 185 .

ZNF185, may help clarify the mechanism underlying its diverse subcellular localization and function (12). The LIM domains are generally cysteine- and histidine-rich domains, 50-60 amino acids in size, sharing double characteristic zinc finger motifs. A diverse group of proteins containing LIM domains has been identified, which displays various functions, including gene regulation, cell fate determination, tumoral formation and cytoskeleton organization. LIM domain proteins were previously shown to be distributed in the nucleus as well as the cytoplasm and exert their functions through interactions with various
Table III. Multivariate analyses using the Cox proportional hazards model.

\begin{tabular}{|c|c|c|}
\hline Variable & Strata & P-value \\
\hline Age (years) & $68.30 \pm 9.26$ & 0.108 \\
\hline Gender & Male, female & 0.967 \\
\hline $\begin{array}{l}\text { Histological type } \\
\text { (adenocarcinoma) }\end{array}$ & $\begin{array}{l}\text { Well, moderate, } \\
\text { poorly differentiated, } \\
\text { mucinous }\end{array}$ & $0.029^{\mathrm{a}}$ \\
\hline T status & $\mathrm{T} 1, \mathrm{~T} 2, \mathrm{~T} 3, \mathrm{~T} 4$ & 0.087 \\
\hline $\mathrm{N}$ status & N0, N1 & 0.268 \\
\hline $\begin{array}{l}\text { Synchronous } \\
\text { liver metastasis }\end{array}$ & Positive, negative & $<0.0001^{\mathrm{b}}$ \\
\hline Lymphatic involvement & Positive, negative & 0.216 \\
\hline Venous involvement & Positive, negative & 0.319 \\
\hline ZNF185 expression & Positive, negative & $0.020^{\mathrm{a}}$ \\
\hline
\end{tabular}

${ }^{\mathrm{a}} \mathrm{P}<0.05$ and ${ }^{\mathrm{b}} \mathrm{P}<0.001 . \mathrm{ZNF} 185$, zinc finger protein 185 .

protein partners (12). Certain LIM domain proteins are known to play a role in the carcinogenic processes. Epithelial protein lost in neoplasm and testin were found to be downregulated in various cancer cell lines $(18,19)$, whereas LIM domain-only protein 4 is considered to be a negative regulator of breast cancer susceptibility gene 1 and promotes breast tumorigenesis $(21,23)$. LIM and SH3 protein 1 was also identified as a promoter of breast cancer, ovarian cancer and hepatocellular carcinoma and is suggested to be the transcriptional target of p53 (25-27).

ZNF185 gene expression was only shown to be downregulated in matched normal tissues from prostate cancer, craniocervical squamous cell carcinoma and non-small-cell lung cancer $(16,17,28,29)$. Vanaja et al $(16)$ reported that the gene expression levels in high-grade (Gleason score 9) prostate cancer cells were suppressed more compared to intermediate grade (Gleason score 6) prostate cancer cells (16). Thus, the dysregulation of ZNF185 gene expression appears to be a frequent event in several cancer types, which suggests its potential role in cancer development. However, there are currently no published reports on ZNF185 in colon cancer. ZNF185 expression was significantly high in well-differentiated adenocarcinoma. In this study, we demonstrated that ZNF185 is a liver metastasis-associated factor, as well as an independent prognosis-deteriorating factor in colon cancer.

Adjuvant chemotherapy is commonly performed to reduce the risk of recurrence and improve the prognosis in patients with colon cancer. According to the National Comprehensive Cancer Network guidelines 2012, all patients with stage III disease should undergo adjuvant chemotherapy. However, stage II patients should also undergo adjuvant chemotherapy when they have high-risk factors, such as T4 lesions, lymphovascular involvement, or poorly differentiated histology (31-33). Liver metastasis is one of the most critical events in the clinical treatment of advanced colon cancer (34). Due to the recent development of clinical studies, certain patients with advanced colon cancer and liver metastasis may become operable. However, the chemotherapeutic regimens for metastatic colon cancer have also improved (35). In our results, ZNF185 
indicated liver metastasis with a sensitivity of $100 \%(19 / 19)$ and a specificity of $13 \%$ (9/68). These high-sensitivity and low-specificity properties are appropriate for a screening test. There is a possibility that adjuvant chemotherapy may be omitted in ZNF185-negative patients. Therefore, ZNF185 may represent a potential prognostic biomarker of colon cancer.

Cancer cell invasion is a multistep process that includes cell attachment, proteolysis of matrix components and cell migration. Hematogenous liver metastasis, in particular, occurs as a consequence of a well-characterized set of sequential events. The detailed properties and functions of ZNF185 in cancerous invasion have not been fully elucidated. We investigated the clinical significance of ZNF185 in the prognosis and treatment of patients with various types of cancer. The results of the present study, which investigated the behavior of ZNF185 in cancerous invasion, may contribute to the development of novel treatment strategies for advanced colon cancer.

\section{Acknowledgements}

The authors would like to thank Tomohisa Machida (Tokai University Hachioji Hospital, Tokyo, Japan) for his technical assistance and helpful discussions.

\section{References}

1. Jemal A, Siegel R, Ward E, et al: Cancer statistics, 2008. CA Cancer J Clin 58: 71-96, 2008.

2. Sadahiro S, Suzuki T, Ishikawa K, et al: Recurrence patterns after curative resection of colorectal cancer in patients followed for a minimum of ten years. Hepatogastroenterology 50: 1362-1366, 2003.

3. Yamada H, Hirano S, Tanaka E, Shichinohe T and Kondo S: Surgical treatment of liver metastases from pancreatic cancer. HPB (Oxford) 8: 85-88, 2006.

4. Suemizu H, Monnai M, Ohnishi Y, Ito M, Tamaoki N and Nakamura M: Identification of a key molecular regulator of liver metastasis in human pancreatic carcinoma using a novel quantitative model of metastasis in NOD/SCID/gammacnull (NOG) mice. Int J Oncol 31: 741-751, 2007.

5. Hamada K, Monnai M, Kawai K, et al: Liver metastasis models of colon cancer for evaluation of drug efficacy using NOD/Shi-scid IL2Rgammanull (NOG) mice. Int J Oncol 32: 153-159, 2008.

6. Matsuyama M, Wakui M, Monnai M, et al: Reduced CD73 expression and its association with altered purine nucleotide metabolism in colorectal cancer cells robustly causing liver metastases. Oncol Lett 1: 431-436, 2010.

7. Matsuo E, Watanabe M, Kuyama $\mathrm{H}$ and Nishimura O: A new strategy for protein biomarker discovery utilizing 2-nitrobenzenesulfenyl (NBS) reagent and its applications to clinical samples. J Chromatogr B Analyt Technol Biomed Life Sci 877 : 2607-2614, 2009.

8. Way JC and Chalfie M: mec-3, a homeobox-containing gene that specifies differentiation of the touch receptor neurons in C.elegans. Cell 54: 5-16, 1988.

9. Freyd G, Kim SK and Horvitz HR: Novel cysteine-rich motif and homeodomain in the product of the Caenorhabditis elegans cell lineage gene lin-11. Nature 344: 876-879, 1990.

10. Karlsson O, Thor S, Norberg T, Ohlsson H and Edlund T: Insulin gene enhancer binding protein Isl-1 is a member of a novel class of proteins containing both a homeo- and a Cys-His domain. Nature 344: 879-882, 1990.

11. Dawid IB, Breen JJ and Toyama R: LIM domains: multiple roles as adapters and functional modifiers in protein interactions Trends Genet 14: 156-162, 1998.

12. Zheng Q and Zhao Y: The diverse biofunctions of LIM domain proteins: determined by subcellular localization and protein-protein interaction. Biol Cell 99: 489-502, 2007.

13. Pérez-Alvarado GC, Miles C, Michelsen JW, et al: Structure of the carboxy-terminal LIM domain from the cysteine rich protein CRP. Nat Struct Biol 1: 388-398, 1994.
14. Schmeichel KL and Beckerle MC: The LIM domain is a modular protein-binding interface. Cell 79: 211-219, 1994.

15. Heiss NS, Gloeckner G, Bächner D, et al: Genomic structure of a novel LIM domain gene (ZNF185) in Xq28 and comparisons with the orthologous murine transcript. Genomics 43: 329-338, 1997.

16. Vanaja DK, Cheville JC, Iturria SJ and Young CY: Transcriptional silencing of zinc finger protein 185 identified by expression profiling is associated with prostate cancer progression. Cancer Res 63: 3877-3882, 2003.

17. Zhang JS, Gong A and Young CY: ZNF185, an actin-cytoskeleton-associated growth inhibitory LIM protein in prostate cancer. Oncogene 26: 111-122, 2007.

18. Maul RS and Chang DD: EPLIN, epithelial protein lost in neoplasm. Oncogene 18: 7838-7841, 1999.

19. Tatarelli C, Linnenbach A, Mimori K and Croce CM: Characterization of the human TESTIN gene localized in the FRA7G region at 7q31.2. Genomics 68: 1-12, 2000.

20. Tobias ES, Hurlstone AF, MacKenzie E, McFarlane R and Black DM: The TES gene at 7q31.1 is methylated in tumours and encodes a novel growth-suppressing LIM domain protein. Oncogene 20: 2844-2853, 2001.

21. Visvader JE, Venter D, Hahm K, et al: The LIM domain gene LMO4 inhibits differentiation of mammary epithelial cells in vitro and is overexpressed in breast cancer. Proc Natl Acad Sci USA 98: 14452-14457, 2001.

22. Song Y, Maul RS, Gerbin CS and Chang DD: Inhibition of anchorage-independent growth of transformed NIH3T3 cells by epithelial protein lost in neoplasm (EPLIN) requires localization of EPLIN to actin cytoskeleton. Mol Biol Cell 13: 1408-1416, 2002.

23. Sum EY, Peng B, Yu X, et al: The LIM domain protein LMO4 interacts with the cofactor CtIP and the tumor suppressor BRCA1 and inhibits BRCA1 activity. J Biol Chem 277: 78497856, 2002.

24. Garvalov BK, Higgins TE, Sutherland JD, et al: The conformational state of Tes regulates its zyxin-dependent recruitment to focal adhesions. J Cell Biol 161: 33-39, 2003.

25. Wang B, Feng P, Xiao Z and Ren EC: LIM and SH3 protein 1 (Lasp1) is a novel p53 transcriptional target involved in hepatocellular carcinoma. J Hepatol 50: 528-537, 2009.

26. Grunewald TG, Kammerer U, Schulze E, et al: Silencing of LASP-1 influences zyxin localization, inhibits proliferation and reduces migration in breast cancer cells. Exp Cell Res 312: 974-982, 2006.

27. Grunewald TG, Kammerer U, Winkler C, et al: Overexpression of LASP-1 mediates migration and proliferation of human ovarian cancer cells and influences zyxin localisation. Br J Cancer 96: 296-305, 2007.

28. Gonzalez HE, Gujrati M, Frederick M, et al: Identification of 9 genes differentially expressed in head and neck squamous cell carcinoma. Arch Otolaryngol Head Neck Surg 129: 754-759, 2003.

29. Medina PP, Carretero J, Ballestar E, et al: Transcriptional targets of the chromatin-remodelling factor SMARCA4/BRG1 in lung cancer cells. Hum Mol Genet 14: 973-982, 2005.

30. Taira M, Evrard JL, Steinmetz A and Dawid IB: Classification of LIM proteins. Trends Genet 11: 431-432, 1995.

31. Benson AB III, Schrag D, Somerfield MR, et al: American Society of Clinical Oncology recommendations on adjuvant chemotherapy for stage II colon cancer. J Clin Oncol 22: 3408-3419, 2004.

32. Staib L, Link KH, Blatz A and Beger HG: Surgery of colorectal cancer: surgical morbidity and five- and ten-year results in 2400 patients - monoinstitutional experience. World J Surg 26: 59-66, 2002.

33. Schiffmann L, Eiken AK, Gock M and Klar E: Is the lymph node ratio superior to the Union for International Cancer Control (UICC) TNM system in prognosis of colon cancer? World J Surg Oncol 11: 79, 2013.

34. Sadahiro S, Suzuki T, Tanaka A, Okada K and Kamata H: Hematogenous metastatic patterns of curatively resected colon cancer were different from those of stage IV and autopsy cases. Jpn J Clin Oncol 43: 444-447, 2013.

35. Baba H, Watanabe M, Okabe H, et al: Upregulation of ERCC1 and DPD expressions after oxaliplatin-based first-line chemotherapy for metastatic colorectal cancer. Br J Cancer 107: 1950-1955, 2012 